\title{
Bessere Kundenorientierung bei der Entwicklung physischer Produkte - Nutzung agiler Vorgehensweisen kombiniert mit Additiven Fertigungsverfahren
}

Philipp Blattert, Werner Engeln

Viele Industrieunternehmen sind auf der Suche nach neuen Strategien für eine zukunftssichernde Produktentwicklung. Die Gründe dafür sind in den Herausforderungen zu suchen, die häufig in schnelle Änderungen von Kundenwünschen, der Verbreitung moderner Informations- und Kommunikationstechnologien, kürzeren Technologielebenszyklen, Forderungen nach ökologischer Nachhaltigkeit wie auch in der weiteren zunehmenden Vernetzung der Wirtschaft zu suchen. Die heutige Entwicklungsumgebung in Unternehmen, mit meist starren Abteilungsstrukturen, wenig Kommunikation mit den Kunden und zwischen den Abteilungen im Unternehmen sowie der Auslieferung eines auf einem einmal erstellen Lastenheft basierenden Produkten wird den Anforderungen nicht mehr gerecht. In diesem Zusammenhang rücken agile Vorgehensweisen gepaart mit additiven Fertigungsverfahren für physische Produkte in den Fokus der Entwicklung.

Keywords: Additive Fertigung, Agile Vorgehensweisen, CoLocation, Selbstorganisierte Teams, Kundenorientierung, Spiralmodell, Produkt- und Prozessinkrement.

\section{Erfordernisse der Produktentwicklung}

Unternehmen müssen sich heute bei der Entwicklung von Produkten vielen Herausforderungen stellen. Dazu zählen Vernetzung der Wirtschaft, Individualisierung und schnelle Änderung von Kundenwünschen, Digitalisierung mit neuen Produkten und Geschäftsmodellen, kürzere Technologielebenszyklen sowie verstärkt dem Thema ökologische Nachhaltigkeit. Die vielfältigen Herausforderungen haben Auswirkungen auf die Planungssicherheit. Unternehmen müssen heute mehr denn je in der Lage sein, schnell auf neue, unvorhersehbare Situationen reagieren zu können.

Aber viele Unternehmen sind immer noch gekennzeichnet durch unflexible Abteilungsstrukturen, schlechte Kommunikation zwischen den Abteilungen, und, was noch 
wichtiger ist, wenig bis hin zu keiner Kommunikation mit den Kunden, während der Entwicklung eines Produktes. Kundenbedürfnisse und damit die Anforderungen sind aber bei vielen Produkten nicht statisch und verändern sich während der Zeit, in der der das Produkt entwickelt wird.

Somit ist es wichtig, während der Produktentwicklung mit den Kunden „im Gespräch" zu bleiben. Nur so kann erkannt werden, ob und wie sich Anforderungen verändern und ob das in der Entwicklung befindliche Produkt noch dem entspricht, was sich die Kunden wünschen. Es reicht bei vielen Produkten einfach nicht mehr aus, vor Beginn des Entwicklungsprojektes ein Lastenheft zu erstellen und dieses dann abzuarbeiten.

Agile Vorgehensweisen sind die Basis für regelmäßige Kundenkommunikation. Sie sind für die Entwicklung von Softwareprodukten schon weitverbreitet. Wie aber lassen sie sich auf physische Produkte übertragen? Es bedarf geeigneter Verfahren zur schnellen und wenig aufwendigen Herstellung von solchen Produkten oder zumindest Produktkomponenten während der Produktentwicklung. Und wie muss ein Entwicklungsprozess gestaltet sein, der die Kundenkommunikation fordert und fördert?

\section{Agile Vorgehensweisen bei der Produktentwicklung}

Im Zusammenhang mit agilem Vorgehen werden häufig einzelne Elemente wie Scrum, Kanban oder Design Thinking genannt und die Einführung eines oder mehrerer dieser Elemente dann damit gleichgesetzt, dass ein Unternehmen agil sei. Dieses wäre aber nur ein Kratzen an der Oberfläche. Agiles Vorgehen taucht viel tiefer in die Organisation ein und beschreibt eine Haltung, die gelebt werden muss. In (Scheller 2017), (Sidky 2015) wird deshalb in agil sein und agil machen unterschieden (Abbildung 1).

Beide Elemente müssen in Unternehmen gegeben sein, um mit Agilität nachhaltig Erfolge zu erzielen. Agil sein bedeutet, die Werte und Prinzipien, welche im Agilen Manifest definiert und festgeschrieben sind (Beck et al. 2002), in die eigenen Handlungsweisen und Denkmuster zu übernehmen. Agil machen liefert die nötige Elemente zur Umsetzung. 


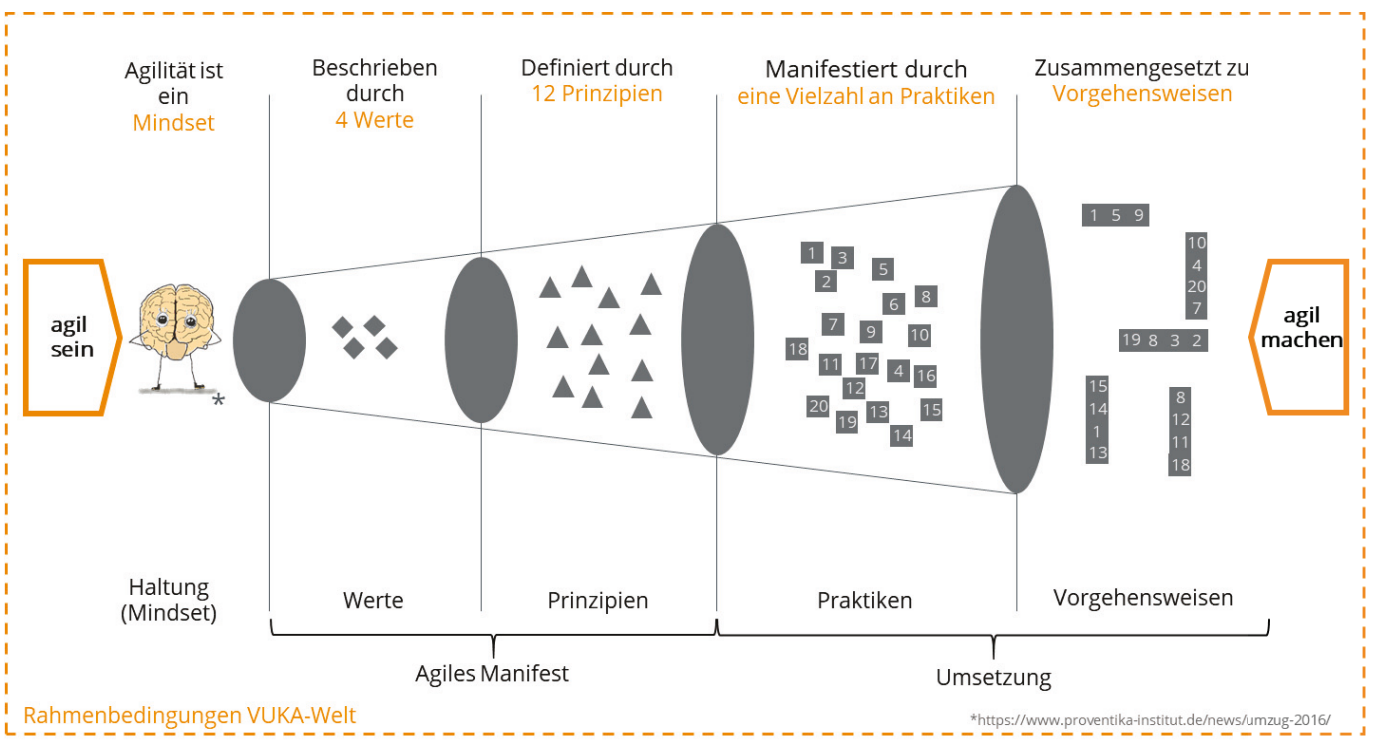

Abbildung 1: Elemente agilen Vorgehens

Aus der praktischen Erfahrung der Einführung von agilen Vorgehensweisen in Unternehmen lassen sich folgende Punkte als Kriterien für den Erfolg herausheben:

— Frühe und regelmäßige Einbeziehung der Kunden in die Produktentwicklung. Anforderungsanalyse durch Kundenbeobachtung und Reviews während der Entwicklung mit Lead-Kunden. Hierbei werden die jeweils, z. B. in Sprints, erarbeiteten Ergebnisse reflektiert und die Akzeptanz des Lösungsansatzes überprüft.

- Ein interdisziplinäres, selbstorganisiertes Team sichert den erfolgreichen Produktentwicklungsfortschritt. Schaffung von Transparenz des Workflows durch Nutzung von Kanban-Boards. Anhand des Kanban-Boards können sich zudem Entscheidungsträger jederzeit über den Projektfortschritt informieren.

- Bereitschaft in der Hierarchie Verantwortung an das Entwicklungsteam abzugeben und auf der anderen Seite die Bereitschaft des Teams, Verantwortung zu übernehmen.

- Das Verständnis im Management, dass agiles Vorgehen nicht bedeutet, jederzeit und kurzfristig neue Projektziele vorgeben zu können. 
- CoLocation, im Lean-Management bekannt unter dem japanischen Begriff Obeya, verbessert und beschleunigt die Zusammenarbeit der Fachexperten im Entwicklungsprojekt durch die Kommunikation von Angesicht zu Angesicht. Ein Projektraum steht dem Entwicklungsteam für den kompletten Projektzeitraum zur Verfügung. Er ist das Kommunikations- und Informationszentrum des Projektes, ausgestattet mit allen benötigten Arbeitsmittel, Anschauungsmaterialien und Modellen.

- Ein ungestörtes Arbeiten des Teams während eines Sprints, sodass sich das Entwicklungsteam zu $100 \%$ auf die Projektaufgabe konzentrieren kann und nicht durch Tagesgeschäft abgelenkt wird. Zeitfenster für Sprints lassen sich, je nach Anzahl und Umfang der Aufgaben, von mehreren Stunden bis zu vier Wochen flexibel gestalten. Im Sinne einer effizienten Entwicklung ist es wichtig, dass die Teams in einen gleichmäßigen Sprintrhythmus gelangen.

- Die Zerlegung des Entwicklungsprojekts in Prozess- oder Produktinkremente ist der Startpunkt agilen Vorgehens. Hierbei hat sich gezeigt, dass bei komplexen Produktentwicklungsprojekten, besonders im Hardwarebereich, eine Zerlegung in Produktinkremente häufig schwierig gestaltet. In diesen Fällen ist es sinnvoll, Prozessinkremente zu definieren, welche vom Team in einem Sprint bearbeitet werden.

Wie unterscheidet sich nun eine agile Vorgehensweise von einem klassischen Vorgehen bei der Entwicklung von Produkten? Bei Unternehmen mit klassischer Vorgehensweise lassen sich folgende Defizite identifizieren:

- Der Kunde wird nicht oder nur zu Beginn der Produktentwicklung einbezogen. Anforderungen werden häufig von den Entwicklungsteams selbst festgelegt. Es gibt nur sehr wenig oder sogar keine Interaktion mit den Kunden.

- Anforderungsprioritäten sind meist unklar. Gibt es im Projektverlauf Anforderungsänderungen oder kommen neue hinzu, so führt das zu Störung im Projekt, was längere Entwicklungszeiten und zwangsläufig fast immer höhere Kosten zur Folge hat.

— Im klassischen Vorgehen werden Projektergebnisse zu einem späten Zeitpunkt und komplett fertigentwickelt ausgeliefert. Änderungen sind dann nicht mehr möglich oder aber sehr teuer. 
- Beim klassischen Vorgehen besitzt ein Entwicklungsteam nur wenige Möglichkeiten der Selbstorganisation. Das Team hat keine oder nur wenig Verantwortung, da Entscheidungen in der Hierarchie getroffen werden.

- Kleinteilig beschriebene Entwicklungsprozesse geben eine starre Vorgehensweise vor. Unvorhergesehene Ereignisse führen zu Störungen, da diese im beschriebenen Prozess nicht berücksichtigt sind.

Diese Defizite können mit Hilfe agilen Vorgehens vermieden werden.

\section{Additive Fertigungsverfahren}

Die Antwort auf die Frage, wie physische Produkte oder Produktelemente schnell während eines Sprints hergestellt werden können, führt zwangsläufig zu den Additiven Fertigungsverfahren. Mit ihrer Hilfe können Entwicklungsteams während eines Sprints Konzept-, Funktions-, Geometriemodelle, technische Prototypen bis hin zu fertigen Produkten schnell herstellen. Dies ermöglicht es, die Iterationszyklen kurz zu gestalten und Kundenreviews noch zielgerichteter durchzuführen, da jetzt ein „begreifbares" Objekt zur Verfügung steht.

In (Atzberger et al. 2020) wird ein in diese Richtung gehender Ansatz, aber mit dem Schwerpunkt Ersatzteile beschrieben. Der Fokus dieses hier vorliegenden Beitrags liegt auf der Verwendung Additiver Fertigungsverfahren in der Produktentwicklung.

Im Vergleich mit anderen Fertigungsverfahren sind die Additiven Fertigungsverfahren noch recht jung. 1951 meldet Otto John Munz sein „Photo-Glyph Recording“ in den USA zum Patent an (Munz). 1981 beschrieb der Japaner Hideo Kodame vom Nagoya Muncipal Industrial Research Center in Japan die Möglichkeit der Herstellung gedruckter Festkörper. Bereits 1984 stellte Charles W. Hull, Gründer der 3D Systems Corporation in South Carolina (USA), den ersten funktionierenden 3D-Drucker her, der mittels Stereolithografie einfache Teile herstellen konnte. 1987 folgte der erste kommerziell erhältliche 3D-Drucker „SLA-1“ (SLA für Stereolithografie), der ab 1988 auf dem freien Markt verkauft wurde. Seit dieser Anfangszeit ist eine Vielzahl unterschiedlicher Verfahren entstanden und kontinuierlich weiterentwickelt worden (Abbildung 2).

Die Gemeinsamkeit der Verfahren liegt darin, dass der Aufbau des Werkstücks schichtweise erfolgt (VDI 3405). Die wesentlichen Unterschiede zwischen den Verfahren sind die eingesetzten Materialien und die dem Schichtaufbau zugrundeliegende Technologie. Die Vorteile der Additiven Fertigungsverfahren bezüglich der Produktgestaltung im Vergleich zu klassischen Fertigungsverfahren sind die: 
- deutlich größere Freiheit bei der Gestaltung von Produkten, Baugruppen und Bauteilen,

- Möglichkeit zur kundenspezifischen Individualisierung der Produkte,

- hohe Funktionsintegration von Produkten, Baugruppen und Bauteilen und damit Einsparung von Montageaufwand.
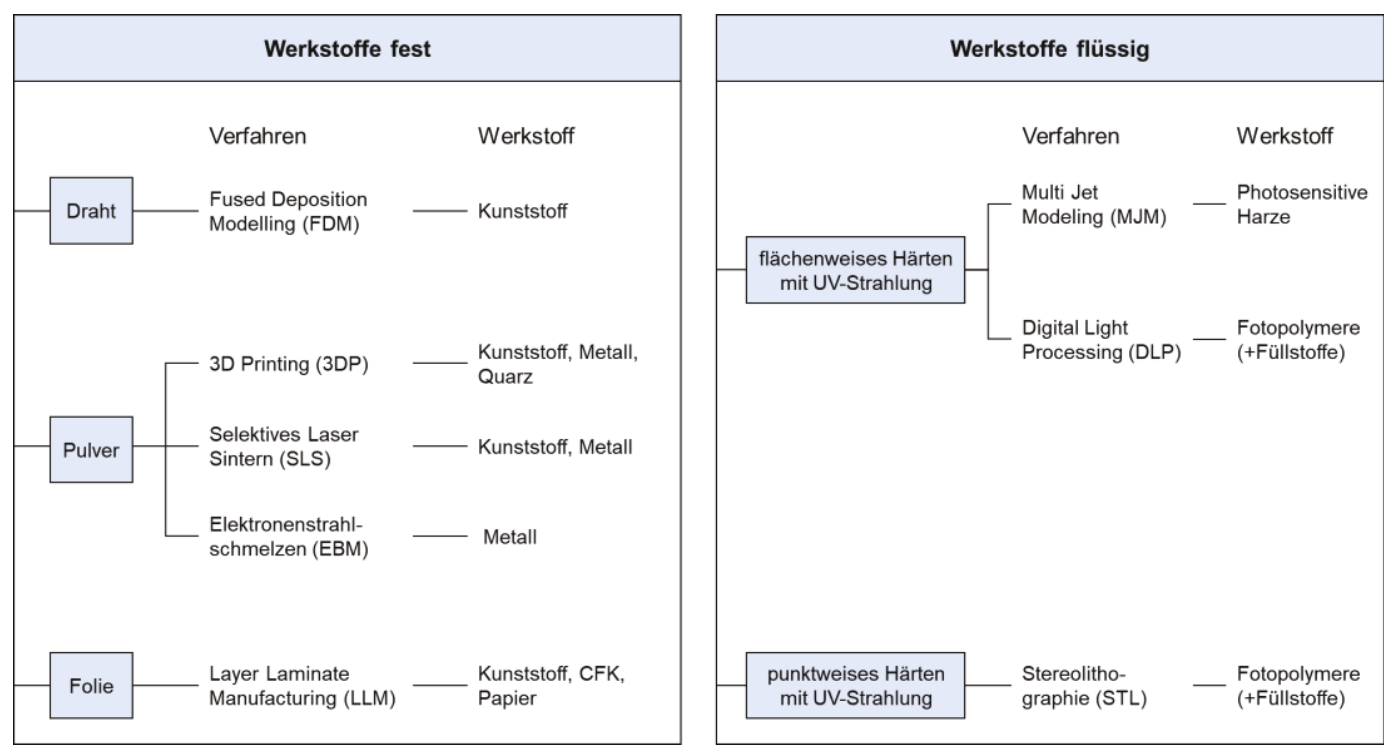

Abbildung 2: Übersicht über die bekanntesten Additiven Fertigungsverfahren

Es fallen viele beschränkende Restriktionen der Gestaltung von Objekten weg, die bei den klassischen Fertigungsverfahren zu beachten sind. Allerdings sind auch Additive Fertigungsverfahren nicht ohne jegliche Restriktionen, es sind auch bei diesen gewissen Einschränkungen zu berücksichtigen (Breuninger et al. 2013), welche aber stark vom jeweiligen Verfahren abhängig sind.

Die genannten Vorteile der Additiven Fertigungsverfahren beziehen sich auf die herzustellenden Objekte. Vorteile für die agile Produktentwicklung ergeben sich besonders aus der einfachen Nutzung der Drucker.

- Zur Herstellung von Objekten - Bauteile, Baugruppen und ganzen Produkten werden keine spezifischen und teuren Werkzeuge benötigt, beispielsweise im Vergleich zu Gießverfahren. Damit sind schnelle und kostengünstige Änderungen am Produkt oder den Bauteilen möglich. 
- Die erforderlichen Druckdaten können sehr einfach aus CAD Daten industrieller CAD Systeme abgeleitet werden. Zudem gibt es eine Vielzahl spezieller, einfach zu bedienender Programme zur Erstellung der Druckdaten. Es sind keine aufwendigen Maschinen-Programme zu erstellen, um dann mithilfe von Werkzeugmaschinen die Bauteile herzustellen.

- In einem Druckvorgang können bei Bedarf mehrere Teile (auch unterschiedliche Teile) gleichzeitig hergestellt werden, was eine bessere Produktivität ermöglicht.

- Die Fertigung komplexer Objekte kann auf einer Maschine erfolgen, ohne Umspannen und ohne Transport von Teilen zwischen unterschiedlichen Maschinen. Es können mehrere Teile in montiertem Zustand gedruckt werden, sodass Montageprozesse entfallen.

- Es handelt sich um einen weitgehend automatischen Fertigungsprozess, der keine Experten zur Maschinenbedienung erfordert. Auch sind die Rüstaufwendungen und Rüstzeiten gering.

- Die Drucker sind weitgehend autarke Betriebsmittel und erfordern wenig Infrastruktur zum Betrieb. Meist reicht ein Stromanschluss.

- Drucker gibt es in sehr unterschiedlichen Ausprägungen sowie Leistungs- und Preisklassen. Häufig sind für Anwendungen in der Produktentwicklung günstige Drucker ausreichend.

- Die Erstellung der Daten und die Erstellung von Prototypen kann räumlich große Distanzen überwinden, wodurch eine globale Vernetzung der Entwicklungsfunktionen möglich ist. Teile für Prototypen oder ganze Prototypen können vor Ort bei Kunden oder sogar von Kunden selbst gedruckt werden und so die Kommunikation mit Kunden vereinfacht und beschleunigt werden.

Eine genaue Beschreibung der Grundlagen und Anwendungen der Additiven Fertigungsverfahren, sich daraus ergebende mögliche neue Geschäftsmodelle wie auch Grenzen und Randbedingungen ihrer Anwendung finden sich beispielsweise in (Breuninger et al. 2013), (Lachmayer et al. 2016), (Gebhardt und Schwarz 2019), oder (Themenheft: 3D-Druck 2017?). Eine regelmäßig aktualisierte Übersicht über die Verfahren der Additiven Fertigung von metallischen Werkstoffen findet sich in (AMPOWER Report 2020). 


\section{Nutzen der Additiven Fertigungsverfahren für eine agile Produkt- entwicklung}

Die Verbesserung der Kundenintegration erfordert einen Entwicklungsprozess, weg von einem Prozess der ausgehend von einem Lastenheft ein Produkt entwickelt, welches dann den Kunden erstmals physisch begegnet, wenn es als fertiges Produkt in den Markt kommt. Das Lastenheft beschreibt die Kundenanforderungen zum Zeitpunkt seiner Erstellung. Durch Änderungen der Kundenanforderungen mit der Zeit aber auch durch im Unternehmen vorherrschende Denkstile (Fleck 2019) und vorgegebene Randbedingungen können Kundenanforderungen und Produkt zum Zeitpunkt der Markteinführung deutlich voneinander abweichen.

Der Entwicklungsprozess muss sich in Richtung eines iterativen Prozesses mit Kundeneinbindung verändern, also einem eher spiralförmigen Entwicklungsprozess (Boehm 1986), (Engeln 2019) Abbildung 3.

Das Zentrum der Entwicklung wird der Zyklus

$$
\text { Entwickeln } \rightarrow \text { Testen } \rightarrow \text { Scheitern } \rightarrow \text { Lernen } \rightarrow \text { Wiederholen }
$$

Die dafür jeweils notwendigen physischen Prototypen werden mit Hilfe Additiver Fertigungsverfahren hergestellt. Diese Prototypen können dann den Kunden vorgestellt und mit diesen diskutiert werden. Da es bei den meisten Produkten nicht möglich sein wird, alle Kunden in diesen Prozess mit einzubeziehen, sollte sich ein Unternehmen in diesem Fall auf seine Lead Kunden konzentrieren.

Die physischen Prototypen erlauben den Kunden eine ganzheitlichere Wahrnehmung des Produktes. Es können die Geometrie, Funktionen und Design von Produktkonzepten direkt für die Kunden und alle an der Produktentwicklung beteiligten Fachabteilungen begreifbar gemacht werden. Der Nutzen daraus ist:

- ein gemeinsames, verbessertes Verständnis für das zu entwickelnde Produkt,

- schnelle Rückmeldungen der Kunden zum Produkt/Produktinkrement als Input für den nächsten Entwicklungssprint und

— schnelles Lernen aus Fehlern für Entwickler:innen. 


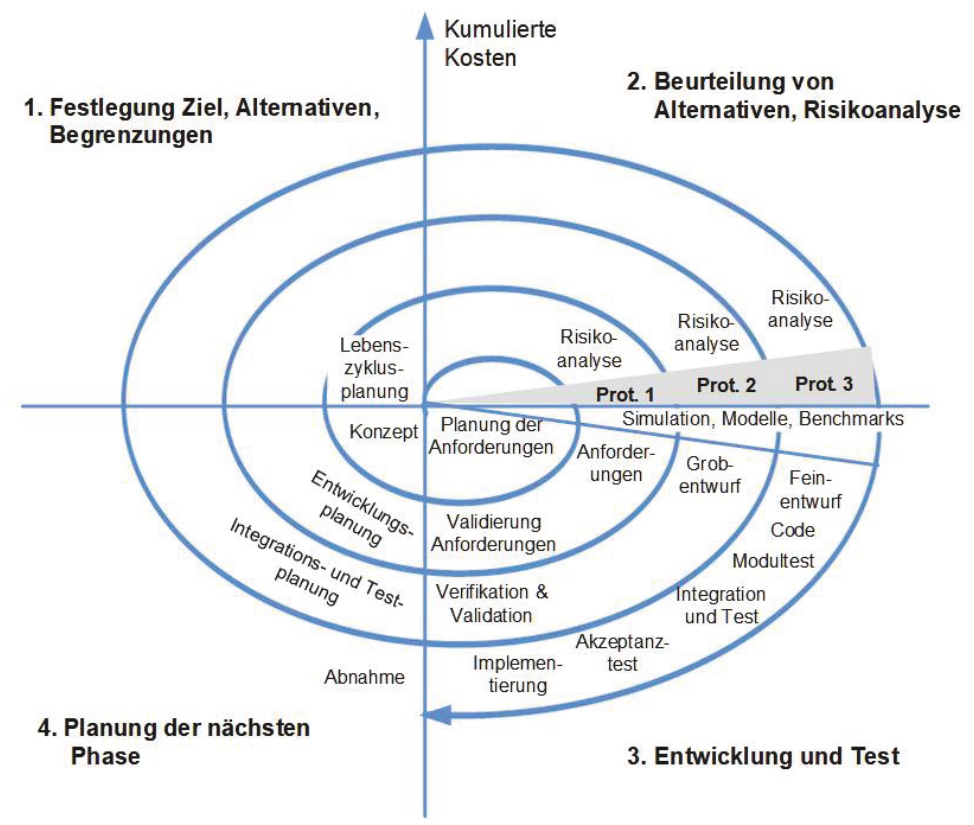

Abbildung 3: Spiralmodell des Entwicklungsprozesses (Engeln 2019)

Weiterhin werden Entwickler:innen animiert, Wissen im Bereich Additiver Fertigung aufzubauen und während des Entwicklungsprozesses zu experimentieren. Hierdurch wird verhindert, dass Lösungskonzepte zu schnell aus einer Meinung heraus ausgeschlossen werden, sondern objektiv durch Ausprobieren aussortiert werden. Die Entwicklungsumgebung im Unternehmen verändert sich hin zu mehr Effizienz, Abbildung 4.

Ein weiterer positiver Effekt ist die Reduzierung des Projektrisikos, da Kunden die Produktentstehung bis hin zum fertigen Produkt iterativ miterleben und mitgestalten können. Das Risiko, dass Produkte bei Markteinführung die Erwartungen der Kunden nicht erfüllen, kann so minimiert werden.

Der Einsatz Additiver Fertigungsverfahren bereits in den Iterationszyklen, ermöglicht es Sprints und Reviews deutlich zu beschleunigen, was wiederum die Gesamtentwicklungszeit verkürzt. Dies verbessert die Wettbewerbsfähigkeit eines Unternehmens und Produktneuheiten können schneller in den Markt gebracht werden.

Additive Fertigungsverfahren sind somit ein wichtiges Werkzeug zur agilen Entwicklung physischer Produkte. 


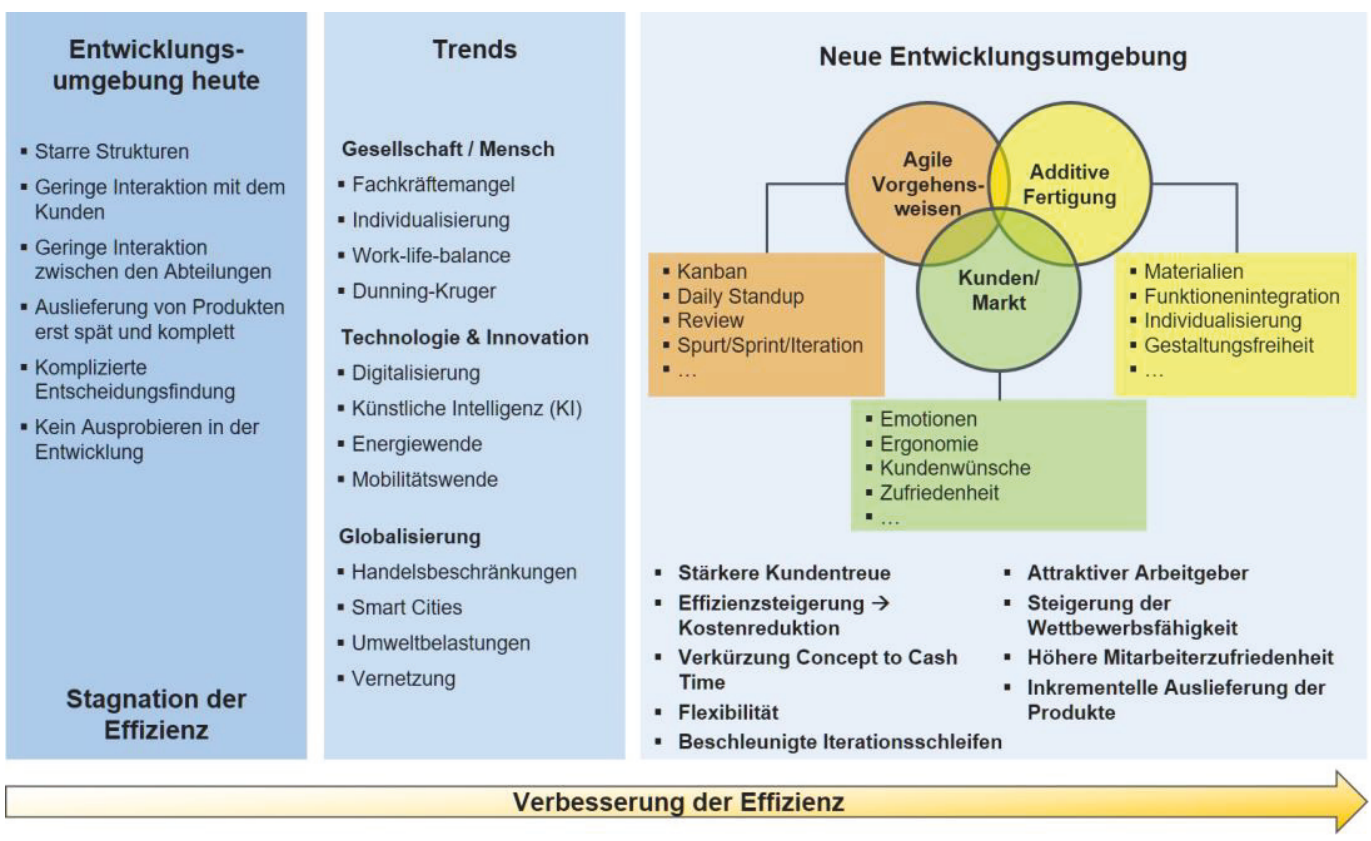

\section{Abbildung 4: Neue Entwicklungsumgebung \\ mit agilen Vorgehensweisen und additiver Fertigung}

\section{Literaturverzeichnis}

VDI 3405, 2014-12: Additive Fertigungsverfahren - Grundlagen, Begriffe, Verfahrensbeschreibung.

AMPOWER Report 2020: Metal Additive Manufacturing Market. Online verfügbar unter https://additive-manufacturingreport.com/, zuletzt geprüft am 23.04.2021.

Atzberger, A.; Bleckmann, M.; Holtmannspötter, J.; Montero, J.; Paetzold, K.; Schmidt, T. S. (2020): Anwendung agiler Entwicklungsprinzipien für die Herstellung von Ersatzteilen mit additiven Fertigungsverfahren. In: Roland Lachmayer, René Bastian Lippert und Stefan Kaierle (Hg.): Konstruktion für die Additive Fertigung 2018. Berlin, [Heidelberg]: Springer Vieweg (Springer eBooks computer science and engineering).

Beck, K.; Beedle, M.; van Bennekum, A.; Cockburn, A.; Cunningham, W.; Fowler, M. et al. (2002): Manifest für Agile Softwareentwicklung. Online verfügbar unter https://agilemanifesto.org/iso/de/manifesto.html, zuletzt geprüft am 23.04.2021.

Boehm, Barry W. (1986): A Spiral Model of Software Development an Enhancement. In: ACM SIGSOFT Software Engineering Notes Volume 11 (Issue 4), S. 14-24.

Breuninger, Jannis; Becker, Ralf; Wolf, Andreas; Rommel, Steve; Verl, Alexander (2013): Generative Fertigung mit Kunststoffen. Konzeption und Konstruktion für Selektives Lasersintern. Berlin, Heidelberg: Springer Berlin Heidelberg. 
Engeln, Werner (2019): Produktentwicklung. Herausforderungen, Organisation, Prozesse, Methoden und Projekte. 1. Auflage. Essen, Deutschland: Vulkan Verlag.

Fleck, Ludwik (2019): Entstehung und Entwicklung einer wissenschaftlichen Tatsache. Einführung in die Lehre vom Denkstil und Denkkollektiv. 12. Auflage. Hg. v. Lothar Schäfer und Thomas Schnelle. Frankfurt am Main: Suhrkamp (Suhrkamp Taschenbuch Wissenschaft, 312).

Gebhardt, A.; Schwarz, A. (2019): Produktgestaltung für die Additive Fertigung. München: Carl Hanser Verlag GmbH \& Co. KG.

Lachmayer, Roland; Lippert, Rene Bastian; Fahlbusch, Thomas (Hg.) (2016): 3D-Druck beleuchtet. Additive Manufacturing auf dem Weg in die Anwendung. Springer-Verlag GmbH. 1. Aufl. 2016. Berlin, Heidelberg: Springer Berlin Heidelberg.

Munz, 0. J.: PHOTO-GLYPE RECORDING. Veröffentlichungsnr: US 2,775,758.

Scheller, Torsten (2017): Auf dem Weg zur agilen Organisation. Wie Sie Ihr Unternehmen dynamischer, flexibler und leistungsfähiger gestalten. 1. Auflage. München: Verlag Franz Vahlen (Vahlen eLibrary: Unternehmensführung, Management, Organisation). Online verfügbar unter https://ebookcentral.proquest.com/lib/gbv/detail.action?doclD=4902342.

Sidky, A. (2015): The secret to achieving sustainable Agility at scale. Online verfügbar unter https://de.slideshare.net/AgileNZ/ahmed-sidky-keynote-agilenz, zuletzt geprüft am 23.03.2021.

Themenheft: 3D-Druck (2017?). Paderborn: Wilhelm Fink (Sprache und Literatur, 46. Jahrgang, 1.-2. Halbjahr (2015) = 115/116).

\section{Kontakt}

Dipl.-Ing. Philipp Blattert

Krehl \& Partner Unternehmensberatung für Produkt + Technik GmbH \& Co. KG Kriegsstraße 113

76135 Karlsruhe www.krehl.com

Prof. Dr.-Ing. Werner Engeln Hochschule Pforzheim

Tiefenbronner Straße 65

75175 Pforzheim

www.hs-Pforzheim.de 